See discussions, stats, and author profiles for this publication at: https://www.researchgate.net/publication/235633013

\title{
The effect of different polymer length on water droplets of reverse AOT
} microemulsion

Article in Physics and Chemistry of Liquids · September 2013

DOI: 10.1080/00319104.2012.760087

CITATIONS

12

4 authors, including:

Soheil Sharifi

Ferdowsi University Of Mashhad

71 PUBLICATIONS 369 CITATIONS

SEE PROFILE

\section{READS}

135

9) $U$ UIm University

21 PUbLICATIONS 148 Citations

SEE PROFILE

Some of the authors of this publication are also working on these related projects:

Mikrorheology for cell mechanics measurements View project

Project Teaching View project 


\title{
The effect of different polymer length on water droplets of reverse AOT microemulsion
}

Soheil Sharifi $^{\mathrm{a}^{*}}$, Othmar Marti ${ }^{\mathrm{b}}$, Sérgio S. Funari ${ }^{\mathrm{c}}$ and Masoud Amirkhani ${ }^{\mathrm{b}^{*}}$

aDepartment of Physics, University of Sistan and Baluchestan, 98135-674 Zahedan, Iran; bInstitut für Experimentelle Physik, Universität Ulm, Albert-Einstein-Allee, 1189081 Ulm, Germany;

'HASYLAB, Notkestrasse 85, D-22607 Hamburg, Germany

*Corresponding authors. Email: sharifi@phys.usb.ac.ir, soheil.sharifi@gmail.com; masoud.amirkhani@uni-ulm.de

\begin{abstract}
:
We study the effect of polyethylene glycol (PEG) on the dynamic and structure of water droplets at the reverse sodium bis-(2-ethylhexyl) sulfosuccinate (AOT) micro- emulsion. The mixture of water and oil with anionic surfactant AOT can form micro- emulsion. The dynamic of microemulsion in the presence of PEG is investigated by photon correlation spectroscopy technique. We mainly focus on the variation of the translational diffusion behaviour as a function of the polymer concentration and polymer length scale. By increasing the content of the lowest PEG length scale $(\mathrm{Mn}=285)$, the dynamic of microemulsion slows down. In addition, one relaxation process is distinguished for all polymer concentration. However, for the two higher polymer length scale $(\mathrm{Mn}=2200$ and 6000), two relaxations are observed and the dynamic of microemulsion speeds up. We used the small angle X-ray scattering technique to monitor the size and the polydispersity of the mixture system (AOT microemulsion/PEG).
\end{abstract}

Keywords: polymer; PEG; microemulsion; light scattering; SAXS; nanophysics

\section{Introduction}

The basic properties of mixed polymers with microemmulsion have received much attention recently $[1,2]$. In general, there are three basic types of microemulsions, direct (oil dispersed in water, o/w), reversed (water dispersed in oil, w/o) and bicontinuous. In this work, we study dynamic and structure of mixture of polyethylene glycol (PEG) on reversed microemulsions and compare it with the direct microemulsion. The behaviour of mixed systems strongly depends on the interaction between the polymer and the surfactant film. For some types of polymers, the polymer-surfactants interaction produces the transient network, which slows down the dynamic of systems [2-4]. Elsewhere, the depletion 
effect was observed in the mixture of non-interacting of polymer with surfactants $[1,5]$. The mixture of water and oil with sodium bis-(2-ethylhexyl) sulfosuccinate (AOT) forms as reversed microemulsion [6-8]. In this work, we study the effect of PEG on AOT microemulsion and compare it with $\mathrm{C}_{12} \mathrm{E}_{5}$ microemulsion mixed with PEG. Ternary $\mathrm{C}_{12} \mathrm{E}_{5}$ microemulsions, consisting of water, decane and non-ionic surfactant, pentaethylene glycol dodecyl ether $\left(\mathrm{C}_{12} \mathrm{E}_{5}\right)$, produce direct microemulsion [1].

The composition of each system is determined by the molar ratio $\mathrm{X}$ of water to surfactant molecules, $\mathrm{X}=\left[\mathrm{H}_{2} \mathrm{O}\right] /[\mathrm{AOT}]$ and the droplet mass fraction $m_{f}=\left(m_{A O T}+m_{H_{2} O}\right) /\left(m_{A O T}+m_{H_{2} O}+m_{D e c}\right)$ which varies by the respective mass of the components water $\left(m_{\mathrm{H}_{2} \mathrm{O}}\right)$, decane $\left(\mathrm{m}_{\text {Dec }}\right)$ and AOT.

In this work, the photon correlation spectroscopy and small-angel X-ray scattering techniques were used to study the collective diffusion coefficient and morphology of mixture of AOT microemulsion/PEG at constant water concentration (molar ratio, $\mathrm{X}=40$ ). The PEG polymer is water-soluble, so, in the mixture of PEG with AOT microemulsion, the PEG can stay inside of water droplet and it can change the diffusion and interaction of droplets.

\section{Experiments}

\section{Materials and preparation}

Sodium-2-diethylhexyl sulfosuccinate, or AOT 99\% (an Alfa product), was dried in the vacuum. Deionised and distilled water is used to prepare the samples for the light scattering and small-angle X-ray scattering (SAXS) measurements. PEG with the different molecular number (285, 2200 and 6000) and n-decane (99\%) were obtained from SigmaAldrich (Munich, Germany). The mixture of AOT microemulsions/PEG was prepared by mixing AOT surfactant, oil (n-decane) and solution of PEG and $\mathrm{H}_{2} \mathrm{O}$. After waiting for several minutes, the samples were single phase and optically clear. The composition of the $\mathrm{AOT} / \mathrm{H}_{2} \mathrm{O} / \mathrm{n}$-decane microemulsion is given by the two parameters water to surfactant molar ratio $(\mathrm{X})$ and water to oil droplet mass fraction $\left(\mathrm{m}_{\mathrm{f}}\right)$. The parameters for explaining the concentration of PEG at microemulsions are shown with the molar ratio of PEG to AOT, $Y$ $=[\mathrm{PEG}] /[\mathrm{AOT}]$. The experimental solutions were prepared by weighting appropriate amounts of AOT dissolved in decane and adding water to the solution.

\section{Methods}

\section{Photon correlation spectroscopy}

Photon correlation spectroscopy measurements were performed on filtered samples using ALV CGS-8f/DLS Instruments Series A6160-V1052 at Ulm University. The light source was a He-Ne laser operating at a wavelength of $632.8 \mathrm{~nm}$ with vertically polarized light. The beam was focused on the sample cell (10-mm glass tubes) through a temperaturecontrolled cylindrical quartz container (two plane-parallel windows), which was filled with a refractive index matching liquid (toluene). The temperature was kept at $20^{\circ} \mathrm{C}$ with an accuracy of $\pm 0.1^{\circ} \mathrm{C}$ during the experiments and the scattering angle $\theta$ was $90^{\circ}$ for all experiments and wave vector calculated by the following equation:

$$
q=\frac{4 \pi n}{\lambda} \sin \left(\frac{\theta}{2}\right)
$$


where $n$ is the refractive index of the solvent, $\lambda$ is the wavelength of the laser or $X$-ray and $\theta$ is the scattering angle. In the photon correlation spectroscopy, the normalised intensity time autocorrelation function $\mathrm{g}_{2}(\mathrm{q}, \mathrm{t})$ was measured [9-11],

$$
g_{2}(q, t)=\frac{\langle I(q, 0) I(q, t)\rangle}{\langle T(q, 0)\rangle^{2}}
$$

where $\mathrm{I}(\mathrm{q}, \mathrm{t})$ is the scattered intensity at a given $\mathrm{q}$ and time $\mathrm{t}$. The $\mathrm{g}_{2}(\mathrm{q}, \mathrm{t})$ function is related to the normalised electrical field correlation function $\mathrm{g}_{1}(\mathrm{q}, \mathrm{t})$ by the Siegert relation assuming that the system is an ergodic media [9-11]

$$
g_{2}(q, t)=1+B\left|g_{1}(q, t)\right|^{2}
$$

where B is the coherence factor of the equipment. All the correlation functions in this work were fitted by summation of two stretched exponential functions. For the high length scale of PEG $\left(\mathrm{M}_{\mathrm{n}}=2200\right.$ and 6000$)$, the correlation function were fitted by two stretched exponential function [9-11];

$$
g_{1}(t)=A \exp \left[-\left(\frac{t}{\tau_{f}}\right)^{\beta_{f}}\right]+(1-A) \exp \left[-\left(\frac{t}{\tau_{s}}\right)^{\beta_{s}}\right]
$$

where $A, \tau_{\mathrm{f}}, \tau_{\mathrm{s}}, \beta_{\mathrm{f}}$ and $\beta_{\mathrm{s}}$ are amplitude of fast relaxation, fast relaxation time, slow relaxation time and the stretched exponential exponent of fast and slow relaxation, respectively. We should note that for the lowest molecular weight of PEG $\left(M_{n}=285\right)$, one stretched exponential is used. The stretched exponential function describes the decay processes that have a distribution of relaxation times $\langle\tau\rangle$. The parameter $\beta(0 \leq \beta \leq 1)$ measures the width of the distribution function. The mean value of the relaxation time is given by

$$
\langle\tau\rangle=\frac{\tau}{\beta} \Gamma\left(\frac{1}{\beta}\right)
$$

where $\Gamma$ is the gamma function. The slow and fast diffusion $\left(D_{s}, D_{f}\right)$ were calculated by $\mathrm{D}=1 / \mathrm{q}^{2}\langle\tau\rangle$.

\section{SAXS experiments}

SAXS experiments using synchrotron radiation are performed at the A2 beam line of the HASYLAB synchrotron facility (DESY, Hamburg) [12-15]. The experiments are done with monochromatic wavelength of $\lambda=1.5 \AA$ and 2D MarCCD165 detector. In the current experiments, the range of scattering vectors is $0.01<\mathrm{q}\left(\AA^{-1}\right)<0.22$, where $\mathrm{q}$ is the length of the wave vector, $q=4 \pi \sin \theta / \lambda$. The measurements are made at $20^{\circ} \mathrm{C}$, and the scattering intensity was corrected for background scattering. The scattering intensity as function of the scattering vector $\mathrm{I}(\mathrm{q})$ of spherical, monodisperse particles can be described with a form factor component $F(q)$, which is proportional to the scattering of a single particle, and a structure factor $S(q)[16]$ :

$$
I(q)=c F^{2}(q) S(q)
$$

c, being a prefactor, which contains the number density of scattering particles and S(q), describes the interaction effect. For the general case of $n$ shells around a spherical droplet core, the form factor reads 


$$
F(q)=4 \pi \sum_{i=0}^{n} \Delta \rho_{i}\left(\frac{\sin \left(q R_{i}\right)-q R_{i} \cos \left(q R_{i}\right)}{q^{3}}\right)
$$

where $R_{i}$ is the radius of the ith shell or, respectively, the core $R_{0}$ and $\Delta \rho_{i}$ is the electron density contrast between the shells, $i$ and $i+1$ with $\rho_{n+1}$ and $\rho_{0}$ being the electron density of the solvent and the core, respectively. So, for a simple core-shell micelle, $n=1$. The structure factor is the Fourier transform of the pair correlation function $g(r)$ :

$$
S(q)=1+4 \pi n \int_{0}^{\infty}(g(r)-1) \cdot r^{2} \frac{\sin (q r)}{q r} d r
$$

The pair correlation function gives the probability to find another particle at a distance $\mathrm{r}$ from the centre of a given particle, relative to the probability to find a particle at this distance in an ideal gas. The pair correlation function is related to the total correlation function $h(r)=$ $\mathrm{g}(\mathrm{r})-1$ and it can be calculated with the Ornstein-Zernike equation [16].

In the case of polydisperse scatterers, it can be shown [16] that if the polydispersity of the particles and their relative distances are uncorrelated, then again a simple expression for the scattered intensity can be obtained by applying appropriate averages in Equation (6):

$$
I(q)=c\left\{\langle|F(q)|\rangle^{2} S(q)+\left\langle|F(q)|^{2}\right\rangle-\langle|F(q)|\rangle^{2}\right\}+I_{b}
$$

where $\langle\ldots\rangle$ denotes an average over a distribution of radii, that is, we only consider size polydispersity, in the SAXS experiment. To calculate the averages, we choose a gamma distribution.

\section{Results and discussions}

In this work, we study the dynamic of droplets of water to the oil in the mixture of PEG with AOT microemulsion. Microemulsions were prepared by mixing AOT with n-decane and water at the constant water to AOT molar ratio $(X=40)$ and constant droplet mass fraction $\left(\mathrm{m}_{\mathrm{f}}=0.1\right)$. Both length scales of polymer and PEG to AOT molar ratios were varied and the autocorrelation function was obtained by the photon correlation spectroscopy. The single diffusive process, which is observed in the neat system and at very low polymer concentrations, splits up into two relaxation modes for the mixtures system (Figure 1). Figure 1 shows an example of the correlation function as a function of polymer concentration at a constant scattering angle of $\theta=90^{\circ}$. This behaviour was observed in a system with two particle size [17,18]: AOT microemulsion with n-octane and heptanes [19] and in the network transition at mixture of PEO-PI-PEO with AOT microemulsion [4]. However, it is not a general point in the mixture system. For example, a single relaxation was observed in the mixture of PEG with $\mathrm{C}_{12} \mathrm{E}_{5}$ microemulsion [1]. In this work, the correlation function was fitted with one stretched exponential functions for the mixture of low polymer length scale $\left(M_{n}=285\right)$ with microemulsion. However, for the mixture of the highest polymer length scale $\left(\mathrm{M}_{\mathrm{n}}=2200\right.$ and 6000) with microemulsion, two exponential function used for fitting. We obtain the relaxation time from Equation (4) and calculated $\left(D_{c}=1 / \tau q^{2}\right)$ the collective diffusion $\left(D_{c}\right)$ for both fast and slow diffusion. The results of the collective diffusion as the function of PEG molar ratio for different length scale of PEG are presented in the Figure 2. For the polymer length scale of $\mathrm{M}_{\mathrm{n}}=285$, the diffusion coefficient of microemulsion shows a decrease upon increasing PEG concentration. But for the two higher molecular weight $\left(M_{n}=\right.$ 2200 and 6000), the fast diffusion increases and slow diffusion decreases with increasing PEG content. Similar behaviour is observed in the network transition of PEO-PI-PEO with AOT microemulsion [4]. When the PEO-PI-PEO is added to the AOT, microemulsion 
elastic forces of the polymer chains in between the droplets (transient network) increase and a displaced droplet returns to its original position on a shorter time scale. The network transition between droplets of the AOT microemulsion can increase the interaction between droplets as it can increase the number of relaxation modes in the network transition. In our case, the PEG cannot produce network between droplets of AOT microemulsion. So, the presence of two relaxations could be attributed to the polymer aggregation in the PEG/AOT microemulsion.

We can summarise the result of light scattering in three different points. First, the fast relaxation increases with length scale of PEG, which can be due to the decreasing repulsive force between droplets [1]. Bellocq found that PEG can reduce the attractions between droplets at $\mathrm{AOT} / \mathrm{H}_{2} \mathrm{O} /$ isooctane [19], which is in agreement with our light scattering results. This effect is similar to adding salt to the AOT microemulsion [8]. Second, the change in the diffusion coefficient upon increasing polymer concentration differs between lowest length scale $\left(M_{n}=285\right)$ and two highest length scales $\left(M_{n}=2200\right.$ and $\left.M_{n}=6000\right)$. For the lowest length scale of PEG added to the AOT microemulsion, the diffusion coefficient of droplets decreases with increasing polymer concentration, whereas for the two other cases with an increase in PEG concentration the diffusion coefficient of droplets increases. Moreover, two relaxation modes are observed for the highest length scale $\left(\mathrm{M}_{\mathrm{n}}=\right.$ 6000). Our results suggest that for the high concentration and length scale of PEG, dissolved polymer leaves the water droplet and aggregate in the oil. The collective diffusion coefficient is obtained by the Stokes-Einstein equation with considering that the hydrodynamic radius of droplets is equal to $8.5 \mathrm{~nm}\left(\mathrm{R}_{\mathrm{H}}=8.5 \mathrm{~nm}\right)$ and viscosity of water at $\mathrm{m}_{\mathrm{f}}=0$ [4], and it shows with the star point at the Figure 2.

As mentioned above, from Figure 3 one sees that for lowest polymer length scale $\left(M_{n}=\right.$ 285) the diffusion coefficient decreases monotonously with increasing PEG con- centration [1]. The reason of the negative slope of Figure 3 can be due to increasing the repulsive interaction between the droplets [1] by increasing polymer concentration. In spite of the same diffusion behaviour for both systems of $\mathrm{C}_{12} \mathrm{E}_{5}$ and AOT micro- emulsion/PEG, we should note that the chief mechanisms are different. For $\mathrm{C}_{12} \mathrm{E}_{5}$-microemulsion/PEG, the depletion effect is a dominated mechanism, which is not the case in the PEG-AOT microemulsion.

The structure of nano-droplets of AOT/ $\mathrm{H}_{2} \mathrm{O} / \mathrm{n}$-decane microemulsion mixed with different concentration and molecular weight of PEG was studied with SAXS experiment at $20^{\circ} \mathrm{C}$. The scattered intensity as a function of $\mathrm{q}$ for AOT/ $\mathrm{H}_{2} \mathrm{O} / \mathrm{n}$-decane microemulsions mixed with PEG is presented in the Figure 4. Our results shows that the size of droplets is constant at $R_{\mathrm{SAXS}}=$ $7.3 \mathrm{~nm}$, with the increase in PEG concentration and change in the length scale of PEG in microemulsion. In general, the size of hydrodynamic radius is bigger than the size of SAXS experiments. This compares with an overall radius obtained by SAXS of $\mathrm{R}_{\mathrm{SAXS}}=\mathrm{R}_{\mathrm{w}}+\mathrm{R}_{\mathrm{AOT}}=$ $7.3 \mathrm{~nm}$, assuming that the thickness of the AOT shell is correctly given by $\mathrm{R}_{\text {Aот }}=1.5 \mathrm{~nm}$.

The structure factor from the SAXS experiment was calculated from Equation (8) by GIFT software [20]. The structure factor shows a peak at $q=0.045 \AA$ (Figure 5); the position of peak is constant with an increase in the concentration and length scale of PEG. The full-width maximum remains unchanged with increasing PEG and length scale of PEG, which indicates a constant order in the system (Figure 5).

In addition, SAXS experiment reveals that the polydispersity is increasing with increasing concentration and length scale of PEG. Several studies indicate that the solubilisation of an adsorbing water-soluble polymer in the water core of an inverse microemulsion changes the flexibility of the interface [21-25], the size of the droplets [24,25] and also the mutual interactions between the droplets [21-25]. We found that with an increase of PEG 
concentration in microemulsion, the $S(q)$ increases (Figure 6). The rate of increase increases with the length scale of PEG (Figure 6).

\section{Conclusions}

The diffusion coefficient of nano-droplets in the mixture of microemulsion with different concentrations and length scale of PEG is studied in this work. By adding PEG to the microemulsion, a single relaxation was observed for the lowest, and for two higher molecular weights, a double-relaxation process was observed. By increasing the polymer concentration, the diffusion coefficient decreased for the polymer length scale of $M_{n}=$ 285. For the two higher PEG length scale $\left(M_{n}=2200\right.$ and 6000), the linear function of the fast and slow relaxation verse PEG molar ratio has positive and negative slopes, respectively. The aggregation of PEG inside the water droplets can produce second relaxation in the system and that with the increase in PEG concentration, the diffusion decreases. The SAXS experiments show that by increasing the concentration and the length scale of PEG, the size of droplets remains constant but the polydispersity increases. Our results show that with increase of PEG concentration the attractive interaction between droplets increases.

\section{References}

[1] S. Sharifi and M. Soud Amirkhani, Soft Nanosci. Lett. 1 (3), 76-80 (2011).

[2] M. Amirkhani, S. Sharifi, and O. Marti, J. Phys. D: Appl. Phys. 45 (36), 365302 (2012). DOI: 10.1080/00319104.2012.737788.

[3] S. Sharifi, M. Amirkhani, M. Reza Mohammadi, M. Aliahmad, and O. Marti, Phys. Chem. Liq. (accepted online Nov. 2012).

[4] T. Blochowicz, C. Gogelein, T. Spehr, M. Müller, and B. Stühn, Phys. Rev. E 76, 41505 (2007).

[5] M. Zackrisson, R. Andersson, and J. Bergenholtz, Langmuir 20, 3080 (2004).

[6] S. Sharifi, P. Kudla, C.L.P. Oliveira, J.S. Pedersen, and J. Bergenholtz, Z. Phys. Chem. 226 (3), 1-18 (2012).

[7] K. Nikjoo, M. Aliahmad, S. Sharifi, and M. Sargazi, Soft Nanosci. Lett. 2 (2), 17-21 (2012).

[8] N. Karimi, S. Sharifi, and M. Aliahma, Opt. Photonics J. 2, 54 (2012).

[9] W. Brown, Dynamic Light Scattering: The Method and Some Applications (Clarendon Press, Oxford, 1993).

[10] M. Amirkhani, S. Volden, K. Zhua, W.R. Glomm, and B. Nyströma, J. Colloid Interface Sci. 328, 20 (2008).

[11] [R. Pecora, Dynamic Light Scattering. Applications of Photon Correlation Spectroscopy (Plenum Press, New York, 1985).

[12] C. Nunes, G. Brezesinski, J.L.F.C. Lima, S. Reis, and M. Lucio, J. Phys. Chem. B 115, 8024 (2011).

[13] C.E. Fernandez, M. Bermudez, A. Alla, S. Munoz-Guerra, E. Tocha, and G.J. Vancso, Polymer 52, 1515 (2011).

[14] S.S. Funari, V. Rebbin, L. Marzorati, and C. di Vitta, Langmuir 27, 8257 (2011).

[15] C. Marco, M. Naffakh, M.A. Gomez, G. Santoro, and G. Ellis, Polym. Compos. 32 (2), 324-333 (2011).

[16] T. Zemb and P. Lindner, Neutrons, X-rays and Light: Scattering Methods Applied to Soft Condensed Matter (Elsevier, Amsterdam, 2002).

[17] A.L. Van Wuyckhuyse, A.W. Willemse, J.C.M. Marijnissen, and B. Scarlett, J. Aerosol Sci. 27 (Supplement 1), 577-578 (1996). 
[18] P.G. Cummins and E.J. Staples, Langmuir 3 (6), 1109 (1987).

[19] A.M. Bellocq, Langmuir 14, 3730-3739 (1998).

[20] G. Fritz and O. Glatter, J. Phys.: Condens. Matter. 18, S2403 (2006).

[21] J. Lal and L. Auvray, J. Phys. II 4, 2119 (1994).

[22] A. Kabalnov, U. Olsson, K. Thuresson, and H. Wennerström, Langmuir 10, 4509 (1994).

[23] M.J. Suarez and J. Lang, J. Phys. Chem. 99, 4626 (1995).

[24] W. Meier, Langmuir 12, 1188 (1996).

[25] M.J. Suarez, H. Levy, and J. Lang, J. Phys. Chem. 97, 9808 (1993) 


\section{Figure Captions}

Figure 1. Autocorrelation functions as a function of relaxation time for a mixture of PEG with AOT- $\mathrm{H}_{2} \mathrm{O}-$ decane at different concentrations and molecular numbers of $\mathrm{PEG}$ at $20^{\circ} \mathrm{C}$.

Figure 2. Collective diffusion coefficient as a function of molar ratio for a mixture of AOT$\mathrm{H}_{2} \mathrm{O}$ - decane, with different length scales of PEG ( $\mathrm{M}_{\mathrm{n}}=285$ cubic, $\mathrm{M}_{\mathrm{n}}=2200$ up-triangle and $\mathrm{M}_{\mathrm{n}}=6000$ down-triangle) of the fast (close points) and slow relaxation processes (open points) at $20^{\circ} \mathrm{C}$. The collective diffusion coefficient is obtained by Stokes-Einstein relation with droplet hydrodynamic radius $\mathrm{R}_{\mathrm{H}}=8.5 \mathrm{~nm}$.

Figure 3. Collective diffusion coefficient as a function of PEG mass fractions of the mixtures of $\mathrm{C}_{12} \mathrm{E}_{5}-\mathrm{H}_{2} \mathrm{O}$-decane at a constant droplet mass fraction $\mathrm{m}_{\mathrm{f}}=0.1$ at temperature $20^{\circ} \mathrm{C}[1]$.

Figure 4. SAXS intensity $\mathrm{I}(\mathrm{q})$ of $\mathrm{AOT} / \mathrm{H}_{2} \mathrm{O} / \mathrm{n}$-decane microemulsion mixed at constant water to the surfactant molar ratio $(\mathrm{X}=40)$ and droplet mass fraction $\left(\mathrm{m}_{\mathrm{f}}=0.1\right)$ mixed with different concentrations and molecular number of PEG at temperature $20^{\circ} \mathrm{C}$. The red lines are hard-sphere polydisperse model.

Figure 5. The structure factor as a function of $\mathrm{q}\left(1 / \mathrm{A}^{0}\right)$ for $\mathrm{AOT} / \mathrm{H}_{2} \mathrm{O} /$ decane microemulsion at constant water to the surfactant molar ratio $(\mathrm{X}=40)$ and droplet mass fraction $\left(\mathrm{m}_{\mathrm{f}}=0.1\right)$ mixed with different concentrations and molecular numbers of PEG at temperature $20^{\circ} \mathrm{C}$.

Figure 6. The size polydispersity at $(\mathrm{q}=0)$ as a function of PEG/AOT molar ratio $(\mathrm{Y})$ for $\mathrm{AOT} / \mathrm{H}_{2} \mathrm{O}$ /decane microemulsion at constant water to the surfactant molar ratio $(\mathrm{X}=40)$ and droplet mass fraction $\left(\mathrm{m}_{\mathrm{f}}=0.1\right)$ mixed with different molecular numbers $(285,2200,6000)$ of PEG at temperature $20^{\circ} \mathrm{C}$. 
Figure 1

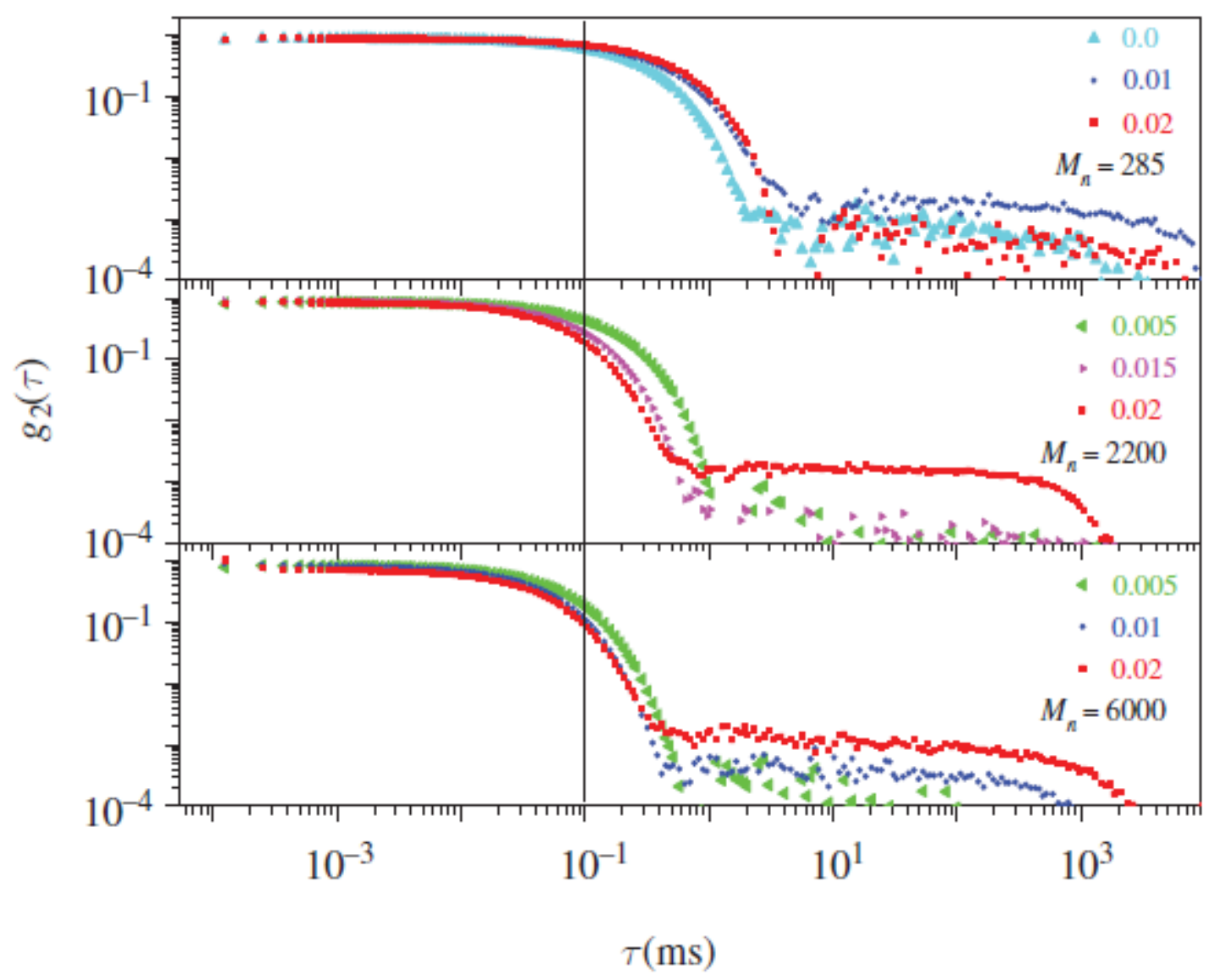


Figure 2

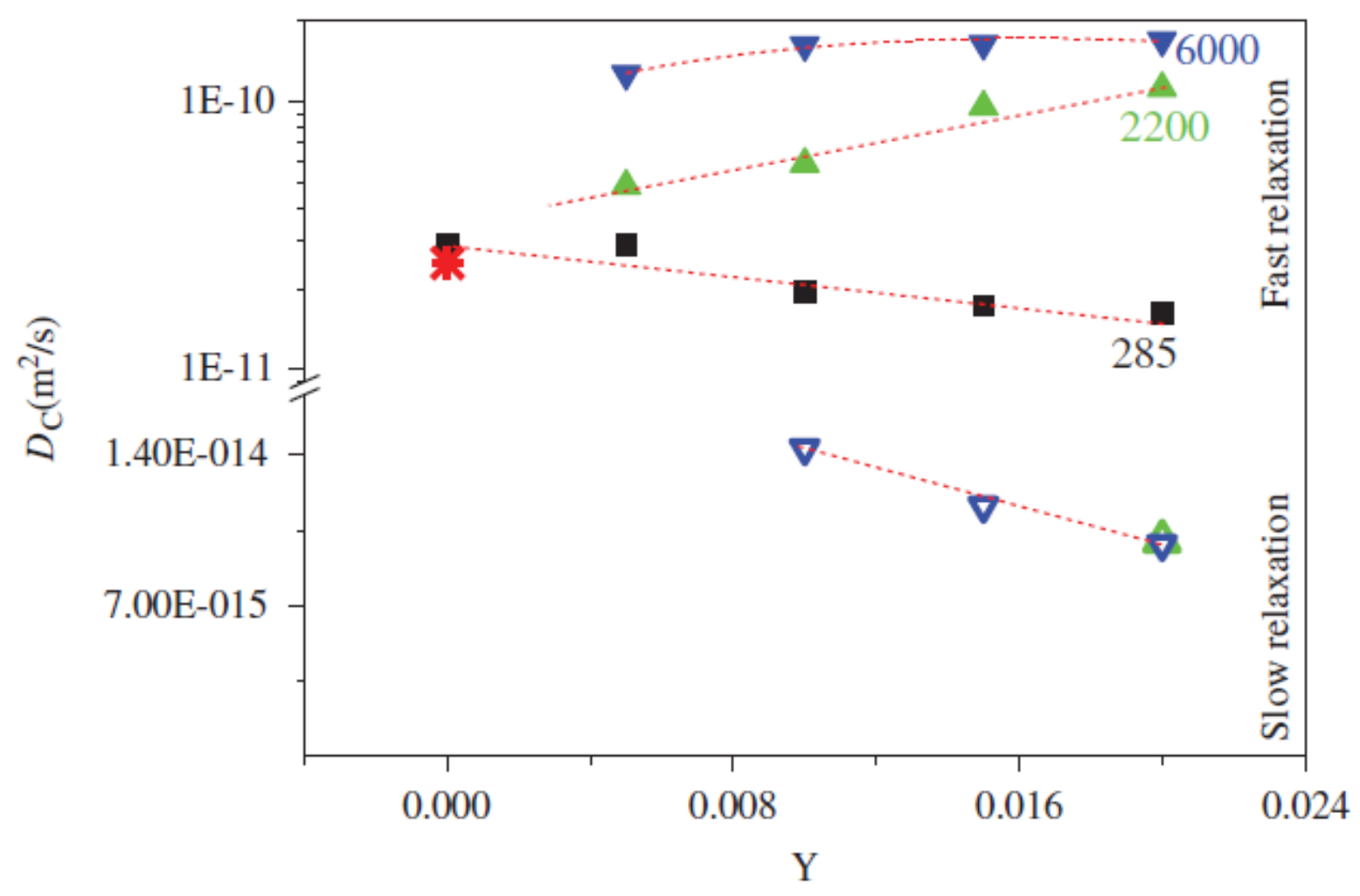




\section{Figure 3}

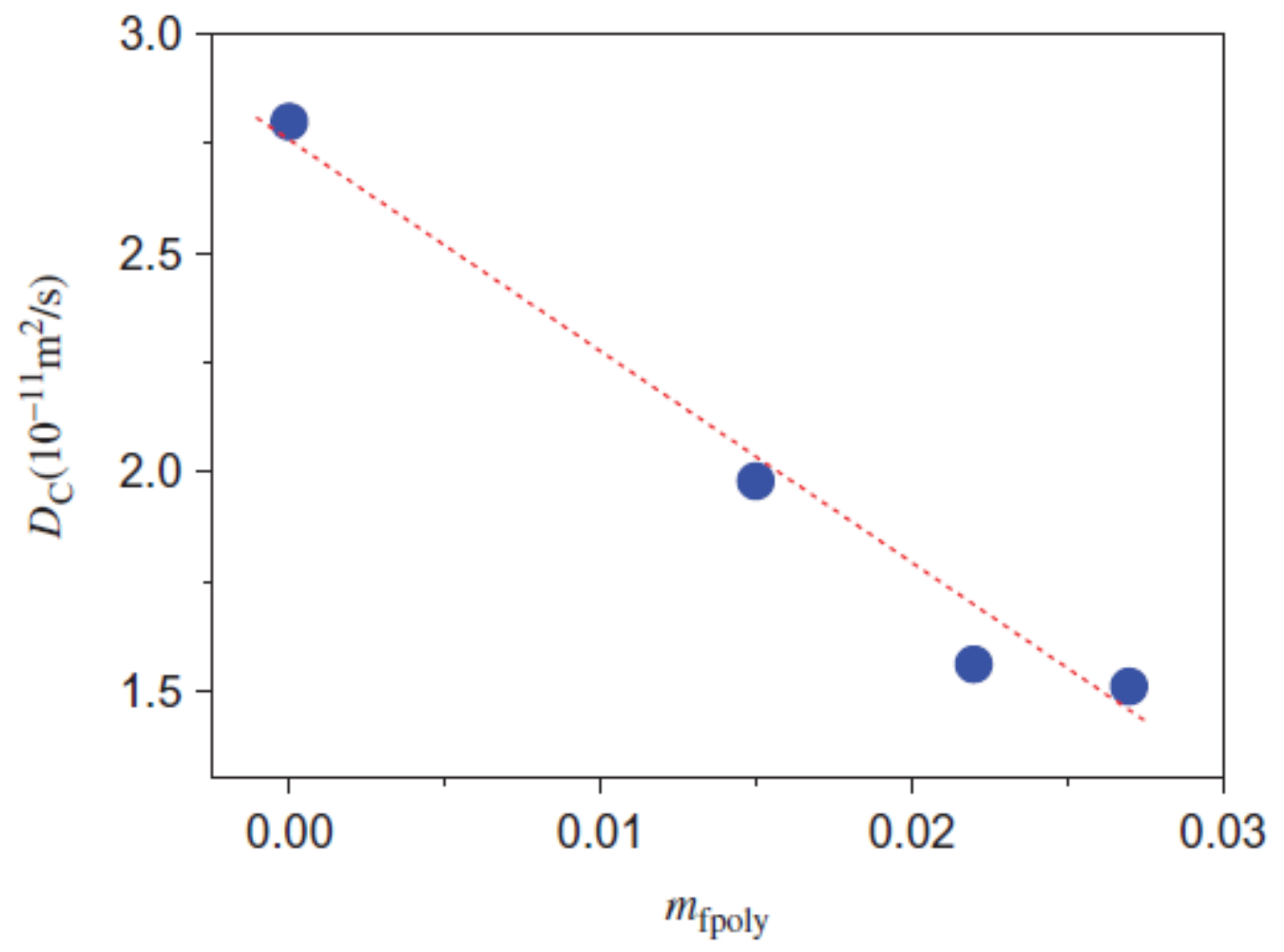


Figure 4

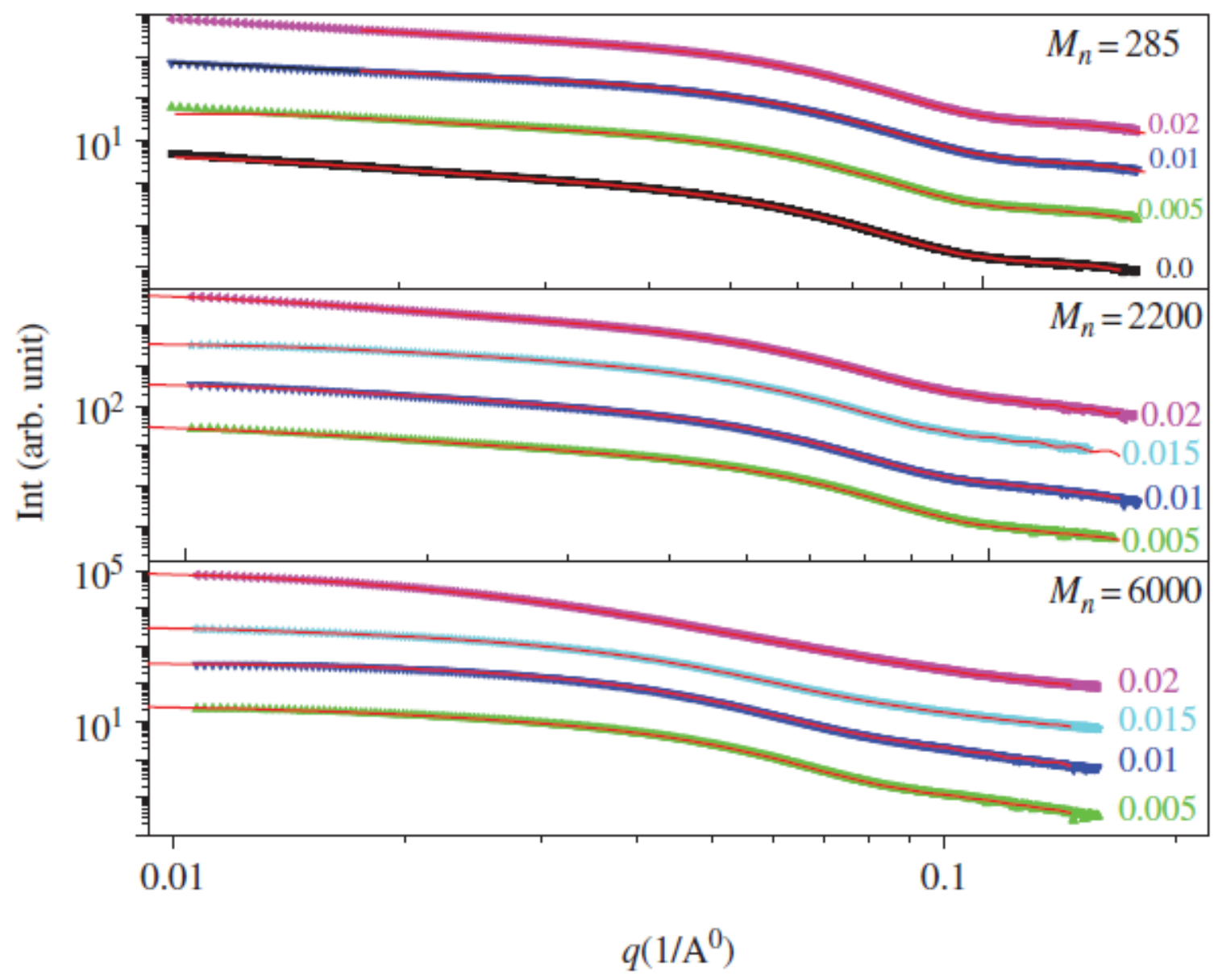


Figure 5

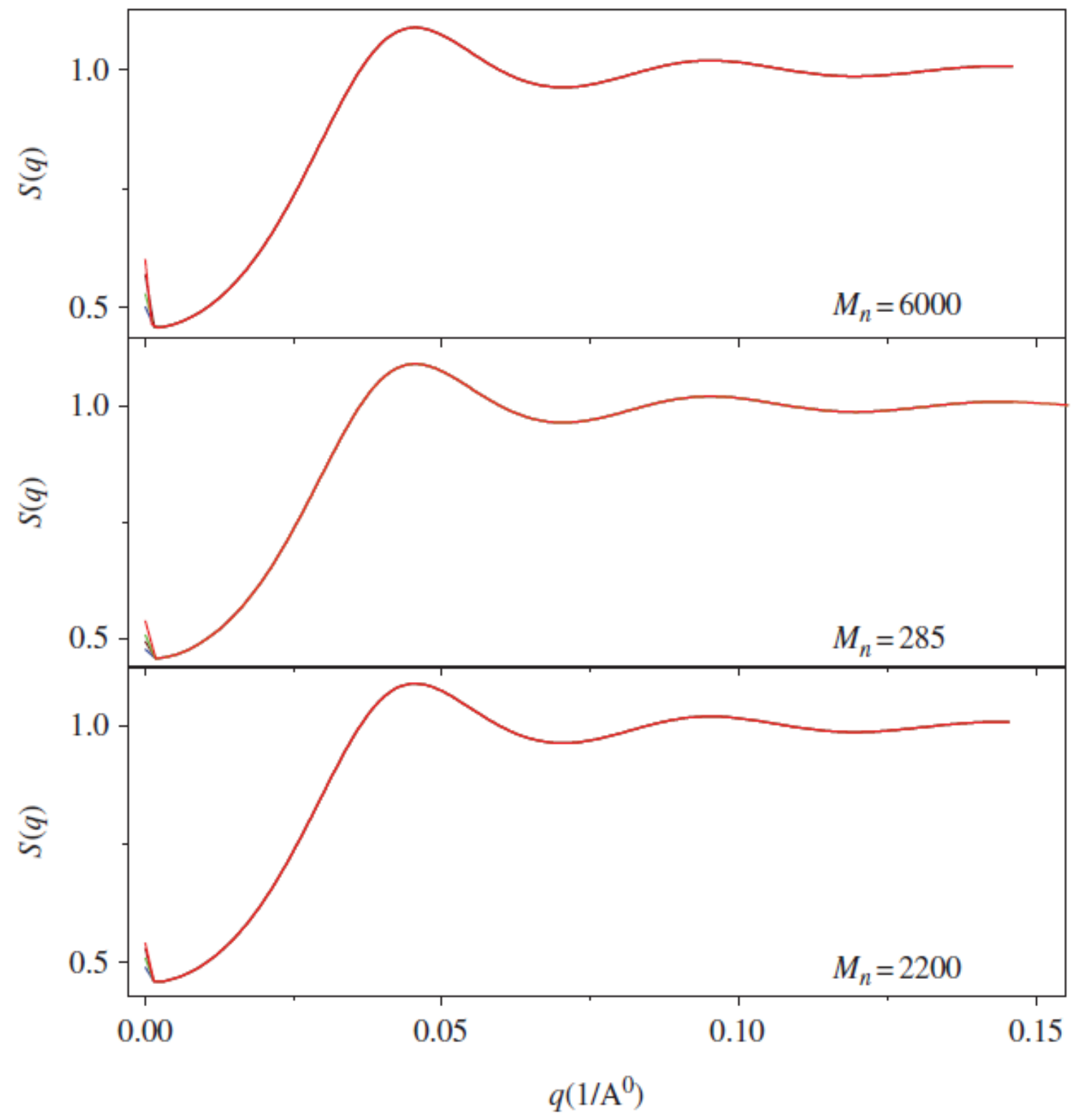


Figure 6

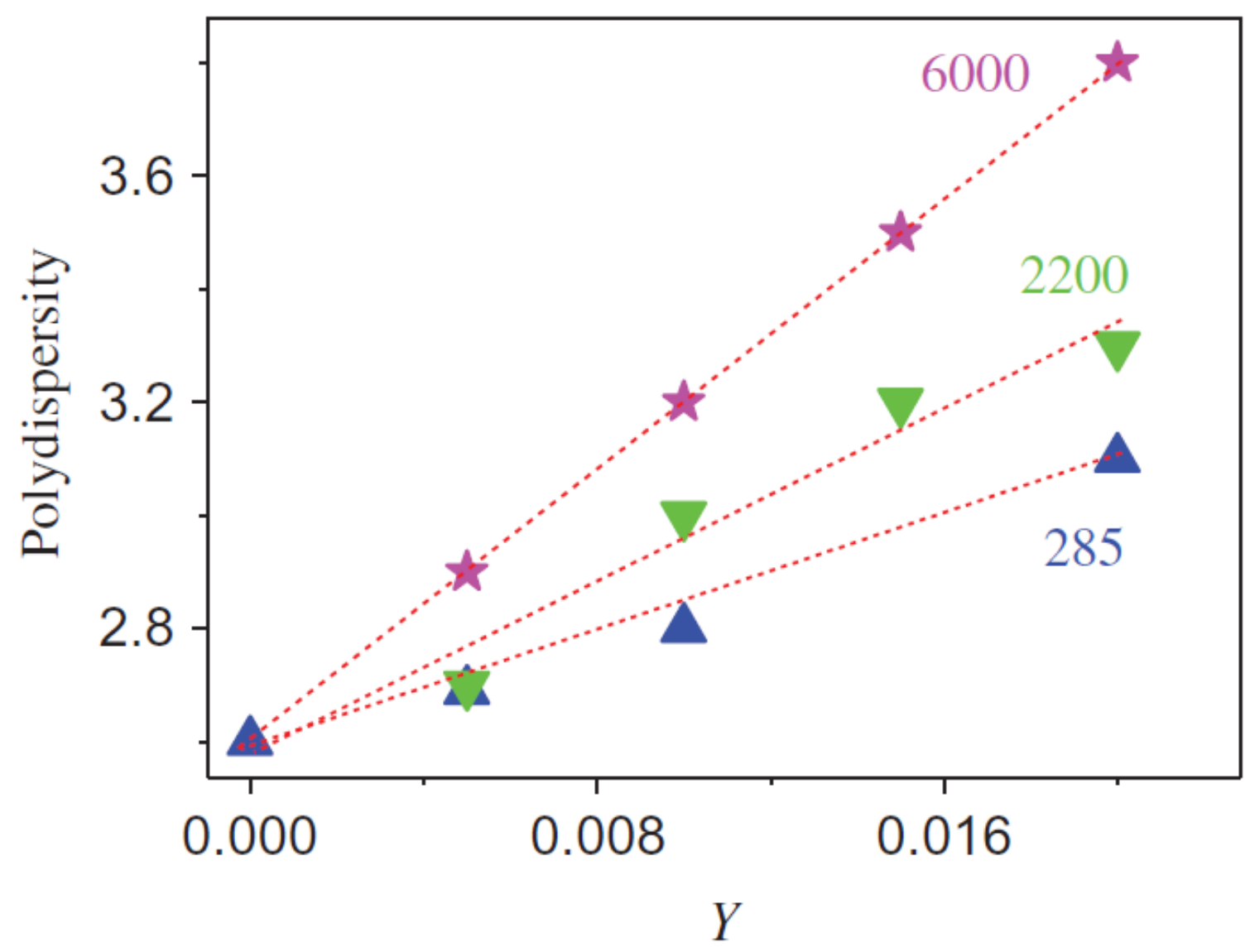

